\title{
LA INEFICACIA CAUSAL DE LO MENTAL Y EL ÉXITO EXPLICATIVO DE LA PSICOLOGÍA
}

\author{
Gustavo Fernández Acevedo \\ Departamento de Filosofía \\ Universidad Nacional de Mar del Plata \\ facevedo@mdp.edu.ar
}

RESUMEN: Las llamadas "estrategias deflacionistas" han constituido una alternativa relativamente popular para enfrentar el problema de la presunta ineficacia causal de los estados mentales en el marco del materialismo no reduccionista. Las propuestas de Lynn Baker y Tyler Burge, desarrolladas en el marco de esta estrategia, coinciden en señalar la necesidad de limitar la importancia de la reflexión metafísica y privilegiar el análisis de la explicación mentalista como fuente para la solución (o "disolución") de los problemas de la causación mental. En contra de esta pretensión, sostenemos que, aun cuando fuera posible reflexionar sobre la causación mental sin recurrir a la metafísica, las dificultades derivadas de lograr una caracterización de lo que constituye una explicación psicológica satisfactoria, sumadas a la competencia explicativa generalizada en la teorización psicológica, resultan obstáculos casi insalvables para estas estrategias.

PALABRAS CLAVE: causación mental, explicación psicológica, metafísica, adecuación explicativa

SUMMARY: The so called "deflationary strategies" have been a relatively popular option for dealing with the problem of the supposed causal inefficacy of mental states in non-reductive materialism. The proposals of both, Lynn Baker and Tyler Burge, developed in the context of this strategy, point out the need to reduce the weight of metaphysical reflection in favor of the analysis of mental explanation as a source to solve (or "dissolve") the problems of mental causation. Contrary to this claim, I hold that, even if reflection about mental causation is possible without metaphysics, the difficulties for these strategies in building a notion of psychological explanation, in addition to the generalised explanatory competence in psychological theorisation, are almost insuperable.

KEY WORDS: mental causation, psychological explanation, metaphysics, explanatory adequacy

\section{El problema de la exclusión causal: ¿solución o disolución?}

El problema de la presunta ineficacia causal de los sucesos/propiedades mentales ha sido uno de los desafíos teóricos más arduos de las últimas décadas para las doctrinas denominadas materialismos (o "fisicalismos") no reduccionistas. Este problema, al cual Kim (1989b, 
1990, 1993, 1998) llamó de la "exclusión causal", ${ }^{1}$ pone a estas doctrinas frente a la indeseable posibilidad de que los estados mentales carezcan de eficacia causal en la producción de otros estados mentales y de la conducta física. Al tratar de abordar el problema, han surgido tentativas de solución de variado cuño, incluyendo propuestas epifenomenistas (Bieri 1992), estrategias del explanandum dual (Marras 1998, 2000) y aun retornos a posiciones dualistas (Lowe 1999), entre otras. $^{2}$

Una estrategia que ha cobrado cierta popularidad en los últimos años frente a la exclusión causal de lo mental es el intentar "disolver" el problema, en vez de resolverlo; esto tiene por objeto mostrar que el problema surge de una manera errónea de concebir cómo debemos desarrollar nuestro conocimiento de lo mental y que, por lo tanto, debería ser desechado. Este tipo de argumentos, denominados por Kim (1998) "argumentos deflacionistas", ha sido defendido en distintas versiones por Baker (1993), Burge (1993), Van Gulick (1993) y, en una variante más reciente, Glymour (1999).

Una de las tácticas que se han empleado con ese propósito ha consistido en mostrar el carácter problemático de los supuestos metafísicos a partir de los cuales se origina el problema, en combinación con la postulación de la necesidad de otorgar prioridad a los aspectos epistémicos de la explicación, dejando de lado la discusión metafísica. Dado que esta clase de respuesta sigue formando parte del menú de opciones teóricas disponibles, resulta de interés examinar sus posibilidades de éxito. En la sección 2, introduciré las versiones de la estrategia deflacionista expuestas por Baker (1993) y Burge (1993). La presentación de los argumentos críticos al marco metafísico será deliberadamente esquemática, ya que mi interés se centra en el aspecto "positivo" de los planteamientos de estos autores. En la tercera sección examinaré distintas interpretaciones posibles de estas posiciones, con el fin de determinar cuál de ellas (realista o irrealista) resulta más consistente con la estrategia elegida por esos autores. Sostendré que el éxito explicativo de la psicología que estas versiones requieren es consistente con una posición (mínimamente) realista. En las secciones 4 y 5 señalaré las dificultades que plantean los aspectos positivos de estas propuestas. En primer lugar, intentaremos mostrar que si bien recurrir a las explicaciones psicológicas proporcionadas

\footnotetext{
${ }^{1}$ Argumentos diferentes de los presentados por Kim, pero que conducen a conclusiones similares respecto de la eficacia causal de lo mental pueden encontrarse en Block 1990, y Jackson y Pettit 1990a.

${ }^{2}$ Cfr. Sabatés 2001 para una presentación amplia de estas alternativas.
} 
por la psicología de sentido común es una opción pertinente para los propósitos de estos autores, no resulta suficiente, pues hace falta examinar las explicaciones de la psicología científica. En segundo lugar, sostendré que los problemas con que nos topamos al tratar de caracterizar lo que constituye una explicación psicológica aceptable y de sostener ciertos supuestos relativos al éxito explicativo de la psicología científica, constituyen dificultades que tornan inviables o, al menos, seriamente cuestionables este tipo de explicaciones.

En síntesis, argumentaré que los aspectos positivos de las estrategias deflacionistas examinadas, independientemente de la evaluación que se haga de sus aspectos críticos, ponen de manifiesto debilidades que dificultan considerarlas como una alternativa promisoria.

\section{Baker, Burge y la prioridad de la explicación sobre la metafísica}

El problema de la causación mental, en opinión de Baker (1993), puede plantearse mediante la siguiente pregunta: ¿cómo pueden las propiedades de contenido de los sucesos internos ser causalmente relevantes para la producción de sucesos conductuales? De la respuesta a esta pregunta se derivará la posibilidad de explicar la conducta en términos de las propiedades de contenido de los estados internos; si tales propiedades son causalmente irrelevantes, y suponiendo ciertas tesis en general admitidas acerca de la naturaleza de la explicación, se sigue que también serán explicativamente irrelevantes. Ahora bien, dado el marco metafísico materialista, ambas preguntas deben ser respondidas de manera desfavorable para el papel causal y explicativo de esas propiedades.

Dos principios, sostiene Baker, constituyen el núcleo del marco metafísico materialista que genera el problema de la causación mental: el principio de clausura causal del mundo físico (CCP) y la noción de superveniencia fuerte $(\mathrm{SS})$ :

(CCP) Toda ejemplificación de una propiedad microfísica que tiene una causa en $t$ tiene una causa microfísica completa en $t$. (p. 79)

(SS) Necesariamente, para toda ejemplificación de cualquier propiedad $F$, hay una ejemplificación de una propiedad microfísica $G, \mathrm{y}$, necesariamente, cualquier cosa que tenga $G$ tiene F. (p. 80)

Ambos principios — observa Baker - no sólo generan un problema para la causación mental, sino que generan también un serio proble- 
ma para la noción de macrocausación, por lo que todas las ciencias especiales e incluso partes de la física resultan afectadas.

El problema de la causación mental se origina cuando notamos que, de cualquier acción, y en virtud de CCP y SS, podemos afirmar que sus determinantes suficientes son propiedades neurofisiológicas, lo cual pone en tela de juicio la pertinencia de afirmaciones como "hizo $x$ en virtud de su deseo de $y$ ". Se necesita, entonces, alguna justificación para admitir afirmaciones de esta última clase; en ausencia de una apropiada, las propiedades mentales parecen ser causalmente irrelevantes.

Con respecto a la macrocausación y a su papel para las ciencias especiales, Baker señala que estos principios implican la idea de que lo que sucede en el micronivel determina todo lo que ocurre en el macronivel; por lo tanto, si toda ejemplificación de cualquier propiedad sobreviene en la ejemplificación de alguna propiedad microfísica, y la ejemplificación de cualquier propiedad microfísica (que tiene una causa) tiene una causa microfísica completa, se necesita una justificación para la afirmación de que las propiedades macrofísicas son causalmente relevantes para algo. En vista de que el marco metafísico establece que la causación es metafísicamente prioritaria sobre la explicación, no puede apelarse al éxito explicativo de las ciencias especiales como forma de obtener esa justificación; dados CCP y SS, el éxito explicativo de tales ciencias es ilusorio.

La conclusión de Baker es que, considerando el marco metafísico que da origen al problema, éste resulta insoluble. Sólo caben dos alternativas ante el problema: o se rechaza una parte de los supuestos metafísicos que configuran el marco a partir del cual surge, o se renuncia casi a la totalidad de las explicaciones que pueden ofrecerse para cualquier suceso, incluyendo las del sentido común y las de las ciencias especiales. Consecuencias tan devastadoras para la ciencia y el sentido común — considera Baker - las convierten en una verdadera reductio ad absurdum del marco metafísico. Su propuesta consiste entonces en tomar como punto de partida filosófico no una doctrina metafísica, sino la explicación:

¿Cómo, entonces, entendemos la causación? Propongo tomar como punto de partida filosófico no una doctrina acerca de la naturaleza de la causación o la realidad, sino un espectro de explicaciones que hemos hallado dignas de aceptación. Este incluye, predominante aunque no exclusivamente, explicaciones científicas. También incluye las explicaciones comunes que encontramos en la vida cotidiana [...]. Construyendo explicaciones como respuestas a preguntas de "por qué", quizás 
con algunas restricciones acerca de lo que se considera una respuesta adecuada, mi propuesta es comenzar con explicaciones que ganen su sustento, más que con la metafísica, la cual me parece gratuita y sólo interfiere con el trabajo real. (Baker 1993, pp. 92-93; las cursivas son mías.)

La causación se convierte, en la propuesta de Baker, en un concepto explicativo; se adopta así una posición muy opuesta a la concepción metafísica que subordina la explicación a la causación. La opción elegida es, entonces, no resolver el problema de la causación mental, sino disolverlo rechazando el marco metafísico a partir del cual se origina.

Baker observa, finalmente, que si bien su propuesta tiene un marcado tono pragmático, no implica una posición antirrealista; no pretende igualar lo que es real con lo que se requiere para formular predicciones y explicaciones. Lo que sí es correcto, a su modo de ver, es que no hay mejor acceso a la realidad que el que se requiere para el éxito cognitivo, interpretado de manera tal que incluya todo aquello que sea cognitivamente necesario para el logro de metas tanto en la ciencia como en la vida cotidiana.

La línea argumentativa seguida por Burge (1993) coincide en varios aspectos importantes con la de Baker. En su opinión, las preocupaciones relacionadas con el presunto epifenomenismo de lo mental son "sintomáticas de un conjunto erróneo de prioridades filosóficas" (p. 97). La causación mental y los poderes causales de lo mental no se pueden comprender concentrándose en las propiedades caracterizadas por las ciencias físicas; por el contrario, nuestro entendimiento de la causación mental deriva primordialmente de nuestra comprensión de las explicaciones mentales, independientemente de nuestro conocimiento de los procesos subyacentes. Si bien Burge considera que deben existir relaciones sistemáticas entre los sucesos mentales y los procesos físicos subyacentes y se inclina por alguna clase de relación amplia de superveniencia (ningún cambio en un estado mental puede tener lugar sin un cambio en los estados físicos), observa que hacer de esto una inferencia favorable hacia el materialismo es una especulación metafísica que ha devenido erróneamente en una especie de lugar común. La base para nuestra comprensión de la causación mental no es, entonces, la metafísica materialista, sino nuestra creencia en la adecuación de nuestras explicaciones causales mentalistas:

Ya que la explicación mentalista produce conocimiento y comprensión, y ya que esa explicación es (a veces) causal, podemos creer firmemente 
que la causación mente-cuerpo es una parte del mundo. La forma básica de entender tal causación es comprendiendo las afirmaciones causales y las explicativas mentalistas en el sentido ordinario, no filosófico, de "comprensión". Cuánto más pueden esclarecer la filosofía o la neuropsicología es algo que está por verse. En cualquier caso, la explicación mentalista y la causación mental no necesitan validación desde la metafísica materialista. (Burge 1993, p. 117)

Pienso que es más natural y fructífero comenzar por suponer, derrotable [defeasible] pero firmemente, que las atribuciones de estados mentales intencionales son centrales en las explicaciones psicológicas de la vida cotidiana y en varias partes de la psicología. Podemos suponer también que los sucesos mentales suelen ser causas y que la explicación psicológica es a menudo una forma de explicación causal. Dados estos supuestos, la "preocupación" relativa al epifenomenismo parece muy remota. (p. 118)

El epifenomenismo, concluye Burge, no constituye una opción metafísica que deba ser tomada en cuenta, con seriedad; al igual que el escepticismo, sería mejor considerarlo un instrumento útil para clarificar nuestras creencias más profundas.

\section{Realismo e instrumentalismo en los argumentos deflacionistas}

Las distintas variantes de las estrategias deflacionistas coinciden en algunos aspectos importantes y difieren en otros. Respecto de los primeros, posiblemente la coincidencia más sobresaliente esté en el diagnóstico: la metafísica materialista no puede constituir la piedra basal para la reflexión acerca de la causación mental. Si los problemas de la causación mental tienen una solución, ésta no provendrá de las doctrinas que asignan una posición de privilegio al mundo físico. Una segunda coincidencia reside en la necesidad de partir de la práctica explicativa, tanto científica cuanto de sentido común. Cabe mencionar, no obstante, que un diagnóstico similar no conduce a todos los autores a sugerir alternativas similares. ${ }^{3}$

\footnotetext{
${ }^{3}$ Van Gulick (1993), dentro del marco de una posición deflacionista, hace del rechazo al supuesto privilegio metafísico de las propiedades y las leyes físicas su argumento principal. Considera que una manera de responder al argumento de la exclusión es poniendo de manifiesto el papel de las leyes y de las explicaciones causales en nuestra economía cognitiva. El papel de las leyes causales es suministrar los medios para formular proyecciones hacia el futuro y formar planes de acción. Si bien las leyes causales ideales para nuestros propósitos explicativos son leyes simples, confiables y precisas, rara vez contamos con leyes de esta clase; en las explicaciones causales, por lo tanto, la precisión a menudo debe ser sacrificada ante las limitaciones pragmáticas acerca de lo que podemos detectar o comprender. Las explicaciones in-
} 
Debido a estas diferencias, la clase de objeciones que deben enfrentar las distintas posiciones deflacionistas varía considerablemente. Es de destacar, no obstante, que estas posiciones a menudo resultan inadecuadas incluso para autores que niegan que el problema de la exclusión causal sea insoluble dentro del marco del materialismo no reduccionista. ${ }^{4}$ Parece haber varias razones por las cuales estas estrategias no son satisfactorias. ${ }^{5}$

tencionales podrán ser menos precisas y confiables que las explicaciones microfísicas de los mismos hechos; sin embargo, existen en la práctica y relacionan propiedades que podemos detectar y que son relevantes para nuestros intereses. Van Gulick intenta mostrar, además, que las propiedades físicas no tienen el estatus especial que usualmente se les atribuye. En su opinión, las leyes y las explicaciones de las ciencias especiales se refieren a patrones de organización de las propiedades de nivel superior que gozan de relativa independencia con respecto a la organización de las propiedades físicas subyacentes. Estos patrones no son menos reales y causalmente potentes que las propiedades y leyes físicas a partir de las cuales se organizan.

Glymour (1999) coincide con Baker y Burge en que la manera metafísica tradicional de comprender la causación mental constituye un error. El problema de la causación mental planteado por Kim y por otros autores, observa, representa un ejemplo acabado de lo que denomina "corriente metafísica principal en filosofía de la mente". En esta corriente, los proyectos filosóficos se encuentran "amurallados" en relación con cualquier uso real de la matemática o la ciencia. Creemos que es verdad, sostiene Glymour, que nuestros pensamientos, creencias, deseos, planes, esperanzas y temores causan nuestras acciones, o al menos causan algo de lo que hacemos. El reto de la causación mental es mostrar cómo es razonable creer en ella. Ante las diversas estrategias que, en su opinión, pueden adoptarse frente a este desafío, Glymour opta por la que denomina "estrategia Humpty Dumpty": si bien el argumento en contra de la causación mental es sólido, por un largo tiempo hemos estado hablando de manera muy satisfactoria sobre los pensamientos como si fuesen causas, y planeamos continuar haciéndolo; la ciencia carece de relación con él. Su posición parece consistir básicamente en sostener que el problema de la causación mental se resolverá en el marco de la investigación científica y no en el de la reflexión filosófica.

${ }^{4}$ Cfr., por ejemplo, Marras 2000.

${ }^{5}$ En opinión de Kim (1998), ninguna de las estrategias "deflacionistas" (entre las cuales incluye las de Baker y Burge) tiene realmente éxito. Si bien considera que los planteamientos de Burge y Baker son correctos al afirmar que "nuestra confianza en la verdad de las explicaciones intencionales familiares excede nuestro compromiso con cualquier principio metafísico recóndito" (1998, p. 61), Kim advierte que nuestras preocupaciones no son evidenciales ni epistemológicas. El problema de la causación mental es, en primer lugar, un problema metafísico: mostrar cómo es posible la causación mental, no si es posible. Al formular la pregunta del cómo, estamos suponiendo que la pregunta de $s i$ ya ha sido respondida afirmativamente. La cuestión es, señala, cómo hacer que nuestra metafísica sea consistente con la causación mental, y la elección que debe realizarse es entre diversas alternativas metafísicas, no entre algunos recónditos principios metafísicos, por una parte, y algunas prácticas o principios epistemológicos apreciados, por la otra. Kim recuerda los prolongados debates en las décadas de 1950 y 1960, respecto de si las explicaciones de 
En los apartados que siguen nos referiremos exclusivamente al aspecto positivo de los análisis de Baker y Burge, esto es, a su apelación a la práctica explicativa tanto científica cuanto de sentido común como base para resolver el problema. Tomando como punto de partida la crítica expuesta por Sabatés (2001), examinaremos previamente la posibilidad de que la estrategia propuesta por estos autores conduzca a una suerte de irrealismo acerca de lo mental; respecto de esto, sostendremos que parece convincente pensar que esa posibilidad es inconsistente con el éxito explicativo de la psicología que esta estrategia requiere.

Sabatés (2001) sugiere que, una vez que se invierte la prioridad causación/explicación, el resultado final será posiblemente un enfoque irrealista acerca de la mente, quizás de tipo retentivo. Pero muchos realistas sostendrían, por el contrario, que en un marco realista la posibilidad de la causación mental es una precondición de la posibilidad de lograr explicaciones psicológicas causales. Sabatés hace notar que, dado el enfoque de la "primacía explicativa" propuesto por Baker y Burge, si bien los predicados mentales podrían ser meras herramientas explicativas y predictivas, la realidad de lo mental no parecería estar entre las tesis que sustentarían. Esto último parece ser consistente con las afirmaciones de Baker relativas a que no intenta igualar lo que existe con lo que resulte necesario para la explicación y la predicción. La realidad de lo mental se podría defender proponiendo una tesis del siguiente tipo: "una propiedad es real si y sólo si figura en explicaciones exitosas"; pero, señala Sabatés, puede suponerse plausiblemente que no es éste el tipo de concepción sobre la realidad de las propiedades que un realista acerca de lo mental quisiera defender. Esta estrategia de solución al problema de la exclusión causal da como resultado una posición en la cual la tesis que se ha de rechazar del materialismo no reduccionista es el realismo acerca de lo mental.

la acción basadas en el esquema creencia-deseo son causales, y señala agudamente que el desacuerdo no es en cuanto a la práctica explicativa, sino a su naturaleza y razón fundamental [rationale]. Estos debates, advierte, son suficientes para mostrar que no puede aislarse fácilmente la práctica explicativa de la comprensión racional de la conducta de las complicaciones metafísicas; la pregunta en torno a si las explicaciones racionalizadoras son una clase de explicación causal involucra temas metafísicos sustantivos. Y aun cuando se haya respondido de una manera favorable a la posición causalista, la metafísica no desaparecerá. Los casos de competencia entre explicaciones de conductas que apelen a sucesos mentales y los que apelen a sucesos neurofisiológicos exigirán tomar posición acerca de la relación entre los sucesos referidos en los respectivos explanantia ( $c f r$. al respecto Kim 1989a y 1994).

Crítica, vol. 37, no. 110 (agosto 2005) 
Si la interpretación que propone este autor es correcta, podría pensarse entonces que la estrategia de la primacía explicativa desemboca en una variante del denominado "instrumentalismo semántico" restringida al ámbito de lo mental. Los predicados mentales sobrevivirían por razones pragmáticas; permitirían la predicción y la explicación, pero no harían referencia a propiedades reales. Ahora bien, el instrumentalismo semántico se ha caracterizado por presentar limitaciones explicativas importantes; las únicas explicaciones que seríamos capaces de ofrecer desde la perspectiva instrumentalista son aquellas que carecen de referencia a entidades teóricas, esto es, aquellas que sólo remiten a entidades o procesos observables. Esta posibilidad no resulta consistente con los propósitos de Baker y Burge de basar la reflexión sobre la causación mental en explicaciones científicas y de sentido común dignas de aceptación, ya que implica una restricción importante del dominio explicativo. Parecería que, pese a las afirmaciones de Baker en sentido contrario, hace falta igualar, en alguna medida, lo que existe con lo necesario para el éxito cognitivo y considerar que los términos mentalistas efectivamente denotan cierta clase de procesos y entidades.

Por otra parte, el instrumentalismo semántico parece ser una caracterización muy poco convincente del conocimiento científico. Si algunos comentadores de esta perspectiva están en lo correcto, ${ }^{6}$ diversas tesis que el instrumentalista se ve obligado a suscribir (entre ellas, la necesidad de concebir la distinción teórico-observacional como una cuestión de clase) constituyen una fuente de dificultades al parecer insalvables.

Parece haber entonces buenos argumentos para no aceptar fácilmente que las posiciones de Baker y Burge puedan ser consideradas irrealistas e instrumentalistas. Supondremos, por lo tanto, la idea de que una interpretación realista del conocimiento y, correlativamente, de la explicación, es una posición más consistente con estas posiciones; sin embargo, el intento de caracterizar la explicación dentro de un marco realista no resulta nada sencillo. Por ahora puede fijarse una condición mínima que parece necesaria para poder afirmar que una explicación es adecuada para una interpretación realista: una explicación que haga referencia a entidades y propiedades en el explanans requiere que tales entidades y propiedades sean reales. ${ }^{7}$ Negar esta condición impediría rechazar explicaciones basadas

\footnotetext{
${ }^{6}$ Cfr. Newton-Smith 1981.

${ }^{7}$ Podría objetarse que en esta discusión estamos suponiendo, sin argumentación, que Baker y Burge deben aceptar el realismo explicativo. No obstante, no parecería
} 
en entidades y propiedades que, según ahora sabemos, son inexistentes, como el éter o el flogisto. ${ }^{8}$ Esta condición, como veremos, es necesaria, pero obviamente no suficiente, para intentar comprender la causación mental partiendo de la comprensión de la explicación psicológica.

Baker y Burge recurren explícitamente a las explicaciones psicológicas como base para la comprensión de la causalidad mental. Por lo tanto, será conveniente profundizar en el examen de algunos aspectos de tales explicaciones. En la discusión siguiente habrá que suponer que estos autores no aceptarían que su posición conduce a consecuencias irrealistas e instrumentalistas; si lo anterior no fuese así, el objetivo de comprender la causación mental a partir de la explicación psicológica se vería drásticamente limitado, considerando la restricción en el dominio explicativo debida al instrumentalismo.

Si la posición defendida por Baker y Burge tiene carácter programático, ${ }^{9}$ puede analizarse la posibilidad de que las explicaciones psicológicas intencionales brinden el fundamento para la comprensión de la causación mental. Es posible plantear entonces la cuestión referente a la importancia relativa de las explicaciones intencionales de sentido común y las explicaciones intencionales científicas en este proyecto. En el caso de Baker, como parece desprenderse de las afirmaciones de la propia autora, ${ }^{10}$ las explicaciones psicológicas científicas son la base principal para la reflexión sobre la causación

consistente defender la realidad de los estados mentales y luego adoptar una posición irrealista con respecto a la explicación que considere a tales estados meramente como ficciones útiles en el contexto explicativo.

${ }^{8}$ Esto no quiere decir que las explicaciones basadas en el éter o en el flogisto no puedan resultar insatisfactorias por otras razones; sin embargo, ninguna razón parece mejor para rechazar una explicación que saber que las entidades o propiedades explicativas a las cuales se hace referencia en el explanans no existen (salvo, quizás, que el explanandum mismo sea falso).

${ }^{9}$ Recordemos las afirmaciones de Baker respecto a tomar como "punto de partida" filosófico el espectro de explicaciones que hemos encontrado dignas de aceptación. Burge, a su vez, considera que "la metafísica materialista no es el punto de partida más convincente para razonar acerca de la causación mente-cuerpo. La práctica explicativa lo es" (1993, p. 118).

${ }^{10}$ Uno de los árbitros de Crítica ha hecho la observación de que Baker no insiste tanto en la explicación psicológica científica o especializada, cuanto en la explicación mentalista propia de la psicología de sentido común. A menos que se acuse de inconsistencia a esta autora, parecería que las afirmaciones destacadas en la sección 2 muestran que, según ella, su propuesta debe ser desarrollada más en el plano de la explicación científica que en el plano de la psicología de sentido común y que, por lo tanto, las observaciones referentes a la explicación científica psicológica resultan pertinentes para examinarla.

Crítica, vol. 37, no. 110 (agosto 2005) 
mental. Esto no es así en el caso de Burge, quien insiste en las explicaciones causales intencionales sin hacer énfasis en las explicaciones intencionales científicas. Por esta razón, resulta pertinente examinar la hipotética suficiencia de las explicaciones intencionales propias del sentido común para lograr el objetivo propuesto por Baker y Burge.

\section{Las explicaciones de la psicología de sentido común}

Recurrir a las explicaciones y predicciones intencionales de la psicología de sentido común como base (al menos parcial) para la comprensión de la causación mental, tal como Baker y Burge lo proponen, plantea una serie de cuestiones complejas. En principio, resulta verosímil pensar que el examen de tales explicaciones y predicciones es indisociable del análisis de otras cuestiones estrechamente vinculadas que versan sobre la naturaleza de la propia psicología de sentido común. Las preguntas que pueden plantearse sobre tal psicología son múltiples, pero parece que no todas ellas, ni las posiciones que se han defendido al respecto, han iluminado nuestra comprensión del fenómeno (Rabossi 2004). Las cuestiones en torno a su naturaleza (esto es, si se trata de una teoría, una red conceptual no sistemática, un conjunto de convicciones básicas sobre nuestro mundo mental y el de nuestros semejantes, etc.) y a su relación con la ciencia en general y con la psicología científica en particular han dado lugar a una amplia gama de respuestas, entre las cuales están la "teoría de la teoría", la "teoría de la simulación", el compatibilismo en la versión fodoriana, y el eliminativismo en sus diversas variantes. La propia caracterización de la psicología de sentido común plantea dificultades teóricas, ya que las caracterizaciones existentes suelen presuponer una toma de posición respecto de ciertas preguntas cruciales para el programa filosófico de quien sugiere la definición, e ignoran hallazgos empíricos en principio pertinentes para una caracterización más precisa. ${ }^{11}$ Supondremos entonces cierta caracterización mínima estándar de la psicología de sentido común, cuyo núcleo está constituido (al menos en una proporción importante) por cierto tipo de estados internos del organismo, las "actitudes proposicionales", estados como "creer", "desear", "temer", etc., a los que se atribuyen poderes causales en

${ }^{11}$ Por ejemplo, Churchland caracteriza la psicología de sentido común como "un cuerpo integrado de saberes populares que conciernen a las relaciones legaliformes que valen entre circunstancias externas, estados internos y conducta pública" (1981, p. 45). La afirmación de que se trata de un cuerpo integrado de conocimiento y que establece relaciones legaliformes resulta importante para los propósitos de Churchland: sostener que se trata de una teoría y luego dar argumentos en favor de su eliminación. 
la producción de las conductas, y con base en los cuales pueden construirse explicaciones causales y predicciones de dichas conductas. Tales explicaciones y predicciones suelen ser consideradas exitosas.

Es posible examinar diversas cuestiones vinculadas con la pretensión de basar la reflexión sobre la causación mental en las explicaciones y predicciones provistas por la psicología de sentido común. Tres de ellas nos interesan en este contexto: si tales explicaciones y predicciones pueden ser suficientes para la reflexión sobre la causación mental; si, en caso de que no lo sean, pueden ser el fundamento principal para tal fin; y, por último, si son filosóficamente no problemáticas. Comenzaremos por esta última cuestión.

No hace falta adherir a alguna clase de eliminativismo con respecto a lo mental para albergar algunas dudas acerca del éxito explicativo de la psicología de sentido común. ${ }^{12}$ El éxito predictivo, al menos parcial, parece innegable; no es tan segura la atribución de éxito explicativo. Admitir el éxito explicativo depende de la aceptación de las premisas teóricas que se atribuyen a la psicología de sentido común, y esto es justamente lo que se pone en tela de juicio en ciertos enfoques filosóficos sobre lo mental. Si no se admite la restricción a la metafísica por la que abogan Baker y Burge, debe aceptarse que las explicaciones y las predicciones proporcionadas por la psicología de sentido común son lógicamente compatibles con posiciones epifenomenistas. Y para ello, en este caso no es necesario que el eliminativismo más radical esté en lo correcto y las entidades postuladas por la psicología de sentido común no existan; tampoco hace falta que tales entidades existan pero carezcan por completo de poderes causales (epifenomenismo), ya que lógicamente es posible la defensa de una posición que sostenga que los sucesos mentales son capaces de causar otros sucesos mentales, aun cuando sean causalmente impotentes en relación con sucesos físicos (posición defendida por Thomasson 1998). Todo lo que hace falta es que tales entidades estén correlacionadas nomológi-

${ }^{12}$ Para una presentación de distintos argumentos en favor del eliminativismo, véanse Churchland 1981, Stich 1983 y Ramsey, Stich y Garon 1990, entre otros; y sobre argumentos contrarios al eliminativismo, véanse Horgan y Woodward 1985, Jackson y Pettit 1990b, O’Brien 1991, Van Gelder 1992 y Forster y Saidel 1994, entre otros. Si el eliminativismo fuese verdadero y afectara (como ocurre en el caso de la propuesta de Churchland) no sólo a la psicología de sentido común, sino también a la psicología científica, la alternativa sugerida por Baker y Burge quedaría descartada casi automáticamente. En tal caso, no sería plausible recurrir a las explicaciones psicológicas dando por sentado que la ontología típicamente supuesta por la psicología no existe. Por esta razón, el rechazo del eliminativismo constituye una condición necesaria para examinar la plausibilidad de las estrategias deflacionistas descritas.

Crítica, vol. 37, no. 110 (agosto 2005) 
camente con estados físicos, los cuales constituirían las causas reales de la conducta observable. Esta correlación nomológica permitiría la formulación de predicciones exitosas, como las que puede ofrecer la psicología de sentido común. En este caso, no obstante, las explicaciones que esta psicología proporciona fallarían por la sencilla razón de que las entidades a las que se atribuye el poder de generar la conducta física carecerían de tal facultad. ${ }^{13}$ Sin embargo, como se expone a continuación, aceptar al menos parcialmente el éxito explicativo resulta necesario para examinar los aspectos positivos de las propuestas de Baker y Burge. En caso contrario, este análisis no sería posible.

El objetivo de basar la reflexión sobre la causación mental en la explicación psicológica de sentido común deberá adoptar un punto de vista conservador respecto de la psicología de sentido común (si bien esto no implica necesariamente la aceptación de la teoría de la teoría). Pero aun cuando se adopte una posición "conservadora" análoga a la de quienes, como Fodor (1987), abogan por una compatibilidad explicativa entre la psicología de sentido común y la psicología científica, no puede aceptarse sin discusión que las explicaciones provistas por la primera constituyan un fundamento suficiente o siquiera principal para la reflexión sobre la causación mental. Pueden ofrecerse distintas razones en favor de esta afirmación.

En primer lugar, a menos que estemos dispuestos a identificar la causación intencional simpliciter con la causación intencional presupuesta por el sentido común y, consecuentemente, la explicación mentalista causal de sentido común con la explicación causal intencional, tendremos que admitir que parte de nuestra comprensión de la causación mental deberá basarse en nuestros análisis de procesos y explicaciones causales intencionales que no forman parte del dominio de la psicología de sentido común. Incluso quienes defienden de manera decidida la compatibilidad de la psicología de sentido común con la psicología científica admiten la existencia de fenómenos intencionales que aquélla no abarca. Horgan y Woodward (1985), por ejemplo, en su defensa de la psicología popular de los ataques eliminativistas de Churchland, aceptan que la psicología de sentido común aparentemente no ha hecho un gran aporte a nuestra comprensión de fenómenos tales como la imaginación creadora o la construcción de imágenes visuales en tres dimensiones; sin embargo, advierten que las teorías

\footnotetext{
${ }^{13}$ Lo expuesto supone, obviamente, que las explicaciones de la psicología de sentido común se conciben como explicaciones causales en las cuales los factores causalmente relevantes son los estados mentales.
} 
basadas en conceptos derivados de la psicología de sentido común, como las teorías de la atribución y de la disonancia cognitiva, son casos notables de conexión entre psicología de sentido común y psicología científica desarrollada. Horgan y Woodward admiten que carecemos de una comprensión proveniente de la psicología de sentido común de diversos fenómenos mentales, y esto plantea claramente la posibilidad de que sí seamos capaces de ofrecer explicaciones de tales fenómenos provenientes de la psicología científica; es plausible suponer, además, que algunas de estas explicaciones postularán relaciones causales.

En segundo lugar, aun admitiendo que las entidades explicativas presupuestas por la psicología de sentido común y las presupuestas por la psicología científica intencional (como es el caso de las teorías de la atribución y de la disonancia cognitiva) compartan rasgos fundamentales, no puede suponerse sin ulteriores análisis que los esquemas explicativos causales utilizados por ambas sean análogos, de modo que basta examinar los primeros para poder establecer conclusiones sobre los segundos.

Por otra parte, y de manera más general, a menos que igualemos lo mental con las actitudes proposicionales propias de la psicología de sentido común, debemos reconocer que existen procesos mentales causales que no involucran los estados intencionales característicos de la psicología de sentido común. La teorización psicológica de las últimas décadas en el campo de la psicología cognitiva "clásica" ha avanzado en el estudio de distintos procesos (lenguaje, percepción y memoria, por citar sólo algunos) que no involucran la causación de estados intencionales de la clase postulada por la psicología de sentido común. Se trataría, en este caso, de estados representacionales en un nivel infrapersonal o subdoxástico frecuentemente inaccesibles a la conciencia que no pueden ser identificados con los estados intencionales propios del sentido común. Sin embargo, las distintas relaciones entre estados/sucesos de esta clase son susceptibles de descripciones funcionales en términos de sus interacciones causales. A menos que neguemos el estatus mental de tales estados, habrá que admitir que dichos procesos pueden iluminar nuestra comprensión de la causación mental. ${ }^{14}$

\footnotetext{
${ }^{14}$ Esto no implica afirmar que la psicología científica no proporcione una taxonomía de los procesos intencionales característicos de la psicología de sentido común, como lo prueban las distintas teorías sobre la atribución causal, las actitudes, las representaciones sociales y la influencia social, esto es, una parte importante de la teorización en el campo de la psicología social contemporánea. Simplemente implica que la taxonomía de los hechos psicológicos característica de la psicología científica
} 
Estas razones parecen bastar, aun para quien concuerde con la defensa de la psicología de sentido común y su compatibilidad con la psicología científica, para no conceder que la explicación intencional del sentido común constituya el fundamento suficiente en el cual basarse para reflexionar sobre la causación mental. Si el dominio explicativo de la psicología científica incluye no sólo los fenómenos característicos de la psicología de sentido común, como de hecho ocurre, sino muchos otros procesos mentales que pueden ser considerados causales plausiblemente, es inevitable concluir que tal reflexión deberá desarrollarse necesariamente también en el plano de la psicología científica. Por otra parte, estas razones también pueden ser consideradas adecuadas para dudar respecto de la posibilidad, más modesta, de que la reflexión sobre la psicología de sentido común constituya el fundamento principal para enfrentar el problema de la causación mental. En efecto, el espectro de los procesos mentales que plausiblemente pueden ser considerados causales incluye, como subconjunto, a los procesos causales característicos de la psicología de sentido común. A menos que el defensor de la estrategia deflacionista proporcione razones para considerar que los procesos contenidos en tal subconjunto son los procesos mentales causales más relevantes (razones que no surgen de tales propuestas), ${ }^{15}$ puede dudarse razonablemente de que el examen de los procesos característicos de la psicología de sentido común constituya la base principal a partir de la cual es posible encontrar una solución al problema de la causación mental.

Si el análisis precedente es correcto y es necesario plantear la reflexión sobre la causación mental en el plano de la explicación psicológica científica, será pertinente examinar algunas cuestiones gnoseológicas que tal tipo de explicación implica; esto es lo que haré en la sección que sigue.

\section{El éxito explicativo de la psicología científica}

Hemos visto que Baker sugiere comprender la causación mental a partir de "un espectro de explicaciones dignas de aceptación", principalmente explicaciones científicas. Burge, por su parte, considera

contemporánea no se agota en el conjunto de procesos y entidades supuestos por la psicología de sentido común.

${ }^{15}$ Conviene recordar, además, que la propia Baker admite que las explicaciones psicológicas científicas deben constituir la parte predominante del conjunto de explicaciones en el cual debe basarse la reflexión sobre la causación mental. Al respecto, véase la nota 10 . 
que la manera adecuada de razonar acerca de la causación mental es partiendo de la práctica explicativa.

Ahora bien, Baker parece advertir las dificultades derivadas de aceptar incondicionalmente cualquier explicación mentalista como base para la comprensión de la causalidad mental, y sugiere, como se recordará, la posibilidad de establecer algunas restricciones acerca de lo que se considera una explicación aceptable. ${ }^{16}$ Burge no propone establecer una restricción similar, aunque es posible suponer que, interrogado sobre la cuestión, estaría de acuerdo en que no toda explicación psicológica puede cumplir la función que pretende asignarle. Una razón trivial para este acuerdo sería sencillamente que no parece factible que todas las explicaciones que encontramos en la psicología sean causales; ${ }^{17}$ una razón más profunda sería que parece muy plausible suponer que no todas las explicaciones son aceptables. Supondremos, entonces, que la necesidad de limitar el espectro de explicaciones que se consideran aceptables es común a las dos propuestas. Esta cualificación aparentemente inocua encierra un problema que echa por tierra buena parte del posible atractivo de su propuesta. Dos interrogantes surgen inmediatamente: en

${ }^{16}$ En una de las muy escasas observaciones respecto de la psicología científica, Baker señala que "para la psicología, nuestra relajada actitud no establece restricciones a priori sobre la taxonomía de la psicología. De hecho, diferentes teorías psicológicas, igualmente bien confirmadas, pueden tener diferentes taxonomías, y así sistematizar y explicar diferentes ámbitos de realidad" (p. 94). Las afirmaciones de Baker son fácilmente aceptables en tanto las diferentes teorías psicológicas no superpongan sus respectivos dominios explicativos. En caso de que esto ocurra, la situación parece ser de competencia explicativa y no puede ser aceptada sin más por quien considere que la unificación y la simplicidad del conocimiento es un desideratum posible.

${ }^{17}$ Cummins (1983) ha observado que muchas teorías científicas no están diseñadas para explicar cambios, sino para explicar propiedades. Estas teorías están destinadas a dar cuenta de las propiedades de un sistema no en el sentido planteado por las preguntas "¿por qué $S$ adquirió $P$ ?" o "¿qué causó que $S$ adquiriera $P$ ?", sino en el sentido que entrañan preguntas como "¿en virtud de qué $S$ tiene $P$ ?" Mientras que la pregunta característica respondida por una teoría transicional es "ipor qué el sistema $S$ cambia del estado $s$-1 al estado $s$-2?", la pregunta característica planteada por una teoría de las propiedades es: “ipara qué tiene la propiedad $S$ el sistema $P$ ?" Para responder a esta última pregunta, la estrategia típica consiste en construir un análisis de $S$ que pueda explicar la posesión de $P$ por parte de $S$ apelando a las propiedades de los componentes de $S$ y su modo de organización. De esta manera, Cummins distingue entre explicación "por subsunción" (bajo una ley causal) y explicación "por análisis" (en partes componentes); mientras que la primera explica cambios, la segunda explica propiedades. En opinión de Cummins, muchos fenómenos psicológicos no pueden ser explicados adecuadamente por medio de explicaciones subsuntivo-causales, mientras que sí pueden serlo mediante explicaciones analíticas. 
primer lugar, ¿cuáles son las restricciones que debemos introducir para considerar que una explicación es adecuada? En segundo lugar, ¿podemos responder a la pregunta anterior sin reflexionar sobre la metafísica de la explicación? Los argumentos de Kim parecen fundamentar sólidamente una respuesta negativa a esta última cuestión; sin embargo, por mor del argumento, supondremos que podemos reflexionar acerca de las restricciones que impondremos a las explicaciones sin hacer metafísica. En lo que sigue nos referiremos exclusivamente a las explicaciones proporcionadas por la psicología científica. $^{18}$ Es conveniente, en consecuencia, esbozar qué es lo que consideraremos como psicología científica. Dos opciones resultan posibles ante esta necesidad: ofrecer ejemplos de teorías psicológicas científicas, o mencionar rasgos comunes a todas las teorías pertenecientes a esa categoría. Proporcionar una caracterización parece más complejo que ofrecer ejemplos de teorías psicológicas científicas; sin embargo, ésta es una impresión algo engañosa, ya que ofrecer tales ejemplos presupone ciertas tesis acerca de lo que es una psicología científica. Por otra parte, la segunda opción enfrenta el riesgo de inconsistencia: si, entre las teorías mencionadas, existen algunas que presuponen principios incompatibles con los de otras, no todas podrán ser ejemplos de buenas teorías psicológicas, ya que algunas de ellas serán presumiblemente falsas. Ahora bien, ante la exigencia, la siguiente afirmación nos parece plausible: una caracterización mínima de lo que hace que una teoría psicológica sea científica deberá incluir el requisito de que sea posible poner a prueba tal teoría a través de los métodos empíricos característicos de la ciencia fáctica contemporánea (experimentos, observación controlada, modelización matemática, etc.) y que sus principios no sean contradictorios con teorías bien confirmadas en campos adyacentes (por ejemplo, los hallazgos de la neuroanatomía y la neurofisiología o la teoría de la evolución). Estos requisitos mínimos parecen suficientes para permitir la inclusión de muchas teorías psicológicas contemporáneas que han merecido la atención de la comunidad científica, y para excluir otras que no parecen ser capaces de satisfacerlos.

Es posible aceptar, como punto de partida, que la existencia de un conjunto de explicaciones satisfactorias en el contexto de una disciplina significa que ésta es capaz de exhibir cierto éxito explicativo; sin embargo (y dejando de lado el espinoso problema de suministrar criterios acerca de la satisfactoriedad de las explicaciones), puede

\footnotetext{
${ }^{18}$ Debo la inclusión de estas consideraciones a las observaciones de uno de los árbitros de Crítica.
} 
plantearse la pregunta relativa a la naturaleza del éxito explicativo. La noción de éxito explicativo de una disciplina (o de una teoría) es compleja incluso en el caso de disciplinas más desarrolladas que la psicología. ${ }^{19}$ Parece plausible la idea de que la teoría de la explicación que se defienda condiciona la posición que se sostenga respecto de la capacidad o el éxito explicativo de una teoría. Si se aplicara el criterio de la identidad estructural entre explicación y predicción, uno de los pilares de la teoría hempeliana de la explicación científica, afirmar el éxito explicativo de una teoría, en principio, no sería un problema. Toda predicción exitosa derivada de la teoría podría haber sido una explicación exitosa, y viceversa. Una teoría que ostente un éxito predictivo notable podría reclamar un éxito explicativo equivalente. Pero si, por alguna razón, el éxito predictivo está desvinculado del éxito explicativo, la situación es muy diferente. ésta es justamente la posibilidad que plantean para la psicología Horgan y Tienson (1990). Según estos autores, las leyes de la psicología intencional, tanto científicas cuanto de sentido común, se caracterizan por presentar excepciones en su propio nivel y por expresar tendencias causales derrotables (soft laws); la tendencia expresada por una ley particular puede ser derrotada o desviada por otra con la que coexiste en un determinado momento. La asimetría entre explicación y predicción está dada por el hecho de que en ocasiones podemos no ser capaces de predecir una conducta debido al desconocimiento de cuál de las combinaciones de tendencias se impondrá (incluso con un conocimiento completo de los estados psicológicos relevantes). Luego de la acción, no obstante, seremos capaces de proporcionar una explicación de ésta apelando a la tendencia que presumiblemente se ha impuesto. ${ }^{20}$ Es necesario ser consciente, sin embargo, de que tal asimetría entre explicación y predicción introduce el riesgo de que una teoría particular logre

\footnotetext{
${ }^{19}$ No carece de interés señalar que, en algunas caracterizaciones de la empresa científica, el éxito explicativo no es un criterio para decidir si una teoría es o no aceptable. Newton-Smith (1981) señala que si bien el objetivo de la ciencia es producir verdades explicativas, "tendemos a prestar más atención a la generación con éxito de nuevas predicciones corroboradas que a la explicación de hechos conocidos, porque, dado un conjunto finito de hechos conocidos, podríamos, con ingenio, concebir alguna teoría — que podría ser muy compleja y embarazosa - a partir de la cual se pudieran derivar esos hechos. Nuestra primera protección contra tales teorías ad hoc consiste en el requisito de que aparezcan nuevas predicciones corroboradas" (p. 243).

${ }^{20}$ Esta puede ser, dicho sea de paso, una buena explicación de la asimetría entre explicación y predicción observada en ciertos ámbitos de la psicología, como la psicología social (por ejemplo, en las primeras tentativas de predecir la conducta con base en las actitudes individuales).
} 
meramente arreglos explicativos post hoc con la finalidad de evitar la refutación, mientras que su capacidad predictiva sea sumamente limitada. El éxito explicativo resulta de esta manera problemático.

Por otra parte, el éxito explicativo, a diferencia del predictivo y el práctico, no parece ser inmune a los cambios teóricos. En la actualidad difícilmente se considerarían satisfactorias las explicaciones de fenómenos físicos proporcionadas a partir de la mecánica newtoniana; sin embargo, para fines prácticos y con ciertas restricciones, puede seguir siendo muy utilizada para la formulación de predicciones. Las nociones de capacidad explicativa y éxito explicativo como propiedades de las teorías científicas incluso han sido puestas en tela de juicio por algunos filósofos de la ciencia; ${ }^{21}$ no obstante, aceptemos provisionalmente que las teorías científicas poseen capacidad explicativa y pueden lograr el éxito en la búsqueda de explicaciones.

Podría afirmarse que la psicología, en su conjunto, tiene éxito explicativo, si se considera la cantidad y la diversidad de fenómenos que son explicados en su marco, fenómenos que carecen de una explicación en el seno de otras disciplinas científicas y en la psicología de sentido común. El éxito explicativo consistiría, entonces, en proporcionar explicaciones de fenómenos que hasta el momento carecen de explicación. Pero tal vez ésta sea una noción demasiado liberal, y posiblemente inapropiada, de éxito explicativo. Éste no puede consistir en una cuestión meramente cuantitativa (esto es, únicamente en términos de la cantidad de explicaciones que se posean acerca de los fenómenos), que no tome en cuenta las características de tales explicaciones. Es posible que un criterio tal esté expuesto a críticas análogas a las realizadas a la propuesta de Laudan (1977) de considerar como problemas científicos legítimos a aquella clase de problemas que no describen estados de cosas reales (por ejemplo, explicar las propiedades y el comportamiento de las serpientes marinas). Como ha observado Newton-Smith (1981), si la verdad de

21 "[L]a explicación científica no es ciencia (pura), sino una aplicación de la ciencia. Uno de los usos de la ciencia es satisfacer algunos de nuestros anhelos; y estos anhelos son muy específicos [...], pero son siempre anhelos de información descriptiva [...]. Por tanto, no puede plantearse en absoluto la cuestión de la capacidad explicativa como tal [...]. Ni puede haber ninguna cuestión acerca del éxito explicativo, como si éste ofreciera una evidencia en favor de la verdad de una teoría que vaya más allá de cualquier evidencia que tengamos a favor de su capacidad de ofrecernos una descripción adecuada de los fenómenos. Porque, en cada caso, un éxito de la explicación es un éxito de la descripción informativa y adecuada. Y si bien es cierto que buscamos explicaciones, el valor de esta búsqueda para la ciencia es que la búsqueda de explicaciones es ipso facto una búsqueda de teorías empíricamente adecuadas y empíricamente fuertes" (van Fraassen, 1980, p. 193). 
los enunciados de los problemas que se desea resolver no desempeña un papel regulador, cualquiera puede elegir arbitrariamente un conjunto de enunciados que considere dignos de aceptación y luego elaborar teorías para resolverlos. La consecuencia sería que la ciencia se convertiría en una serie de conjuntos de problemas discrecionalmente elegidos y de teorías erigidas para resolverlos, algunas de las cuales deberían ser evaluadas con la misma aceptación que otras con base en su capacidad para resolver sus propios problemas. El modelo de Laudan convierte a la ciencia, de esta manera, en un absurdo. $^{22}$

Suponer que una ciencia (para este caso, la psicología) tiene éxito explicativo solamente porque proporciona explicaciones a muchos problemas, sin examinar la legitimidad de tales problemas (y de las explicaciones correspondientes), parece conducir a las mismas consecuencias indeseables. Por ejemplo, aplicando este criterio resultaría difícil negar que el psicoanálisis, pese a las conocidas objeciones teóricas y metodológicas que se han presentado en su contra, tiene mayor éxito explicativo que la mayoría de las teorías rivales (si no todas) en su dominio. La situación puede ser peor aún si se admiten explicaciones de fenómenos teóricos. Una teoría compleja, que postule muchas entidades teóricas y presuntas relaciones objetivas entre tales entidades, puede aventajar claramente a sus rivales en el número de explicaciones que proporciona, simplemente por el hecho de que propone más entidades y relaciones que sus rivales, aun cuando muchas de estas entidades puedan resultar a la postre ficticias. ${ }^{23} \mathrm{La}$ teoría más explicativa podría revelarse, en última instancia, como la más insatisfactoria. Parece claro que una noción plausible de éxito explicativo debe descartar esta posibilidad.

Una primera restricción obvia para determinar un criterio de éxito explicativo es, entonces, que los explananda del conjunto de explicaciones que fundan la atribución de éxito explicativo de una disciplina hagan referencia a estados de cosas reales. Sin embargo, aquí entra

\footnotetext{
${ }^{22}$ También parece pertinente la observación de que, según esta metodología, “una teoría que resuelva muchos seudoproblemas puede ser mejor que una que resuelva problemas reales, pero en menor cantidad; las teorías que resulten ser las mejores pueden proporcionarnos menos conocimiento del mundo que las peores. Siendo esto así no se entiende, entre otras cosas, por qué [Laudan] dice que en la historia de la ciencia ha habido progreso cognoscitivo" (Comesaña 1995, p. 27; las cursivas son del autor).

${ }^{23}$ La postulación, por parte del psicoanálisis, de los instintos de vida y de muerte y de sus interacciones como principios explicativos fundamentales de una vasta serie de fenómenos podría ser un ejemplo apropiado de esta posibilidad.
}

Crítica, vol. 37, no. 110 (agosto 2005) 
en juego una segunda dificultad: la competencia explicativa. El hecho de que existan diferentes entidades y propiedades explicativas no se debe solamente a la amplitud del espectro de fenómenos que se han de explicar, sino también a la coexistencia de teorías explicativas sobre esos mismos explananda, que proponen entidades, propiedades y leyes (en caso de que las haya) diferentes como base para la explicación. Tales teorías, además, no compartirán los mismos criterios acerca de lo que constituye una explicación adecuada.

Suponer que podemos seleccionar, dentro del conjunto de las explicaciones provistas por la psicología, una gama de ellas que consideramos "dignas de aceptación" es suponer (razonablemente) que esta disciplina ha tenido cierto éxito explicativo; es decir, ha logrado explicar satisfactoriamente (y causalmente, para los intereses de Baker y Burge) un espectro suficientemente amplio de hechos. No sólo debemos suponer que ciertas teorías han tenido éxito explicativo; debido a que la psicología se ha caracterizado por la coexistencia de diversas teorías sobre muchos fenómenos, y no hay un sistema teórico que provea explicaciones causales satisfactorias de conjuntos muy amplios de hechos, es plausible sospechar que las explicaciones causales satisfactorias deberán extraerse de diversas teorías. Es razonable suponer que el éxito explicativo de una disciplina no es idéntico al éxito explicativo de las teorías vigentes, aunque depende de él. Ahora bien, parece indudable que la posibilidad de atribuir éxito explicativo puede diferir de manera notable de una disciplina a otra. Una evaluación favorable del éxito explicativo será mucho más probable en aquellos casos en los que exista consenso respecto de la mejor teoría explicativa disponible, que en los casos en que se disponga de alternativas teóricas en competencia y de méritos comparables. Parece plausible describir la competencia explicativa, dentro de la psicología, como un fenómeno que se plantea en varios niveles. Puede observarse competencia explicativa en un nivel que podríamos denominar "macro", esto es, de competencia entre lo que genéricamente se podrían caracterizar como "paradigmas" o "programas de investigación". Este tipo de competencia teórica se ilustra en los debates actuales entre los partidarios de la psicología evolucionista y los defensores del (así llamado por los primeros) "modelo estándar de las ciencias sociales". ${ }^{24}$ En un nivel intermedio (aunque es muy

\footnotetext{
${ }^{24}$ En este nivel no es inusual la mención a la competencia entre el conductismo y el cognitivismo. Con todo, puede objetarse que no existe competencia en este nivel debido a que lo que en realidad se produjo fue una transición entre paradigmas o programas, y no una coexistencia.
} 
difícil negar la posibilidad de que pueda ser considerado también un nivel "macro") entraría la competencia explicativa, dentro de la psicología cognitiva, entre, por ejemplo, el conexionismo y los enfoques simbólicos. Por último, en un nivel que podríamos llamar "micro" se encuentra la competencia entre teorías de alcance limitado sobre fenómenos "locales". Ejemplo de esto podrían ser, dentro del campo de la psicología social, las teorías que intentan explicar la coherencia actitudinal (teoría de las actitudes basada en el equilibrio, de F. Heider; teoría de la resistencia de las actitudes, de Osgood; teoría de la disonancia cognitiva, de L. Festinger). Estos niveles se pueden caracterizar de acuerdo con el grado de coincidencias que mantienen los enfoques competitivos. La competencia explicativa plantea, cabe suponer, un problema para la pretensión de que una disciplina ha logrado globalmente éxito explicativo. ${ }^{25} \mathrm{Si}$ bien la coexistencia de explicaciones se tolera en ciertos casos específicos, la sobreabundancia sistemática de explicaciones se considera problemática para nuestra economía cognitiva.

Quienes deseen seguir la propuesta de Baker y Burge se encontrarán con una tarea ímproba por delante: deberían proponer criterios teóricamente neutrales acerca de lo que constituye una explicación psicológica causal adecuada, que permitan seleccionar, del conjunto de teorías psicológicas disponibles, aquellas explicaciones causales satisfactorias. Estos criterios, a su vez, no deberán incluir consideraciones metafísicas relativas a la legitimidad de la explicación, ya que, como hemos visto, su propuesta pretende despojar a la metafísica de su lugar de preeminencia en el análisis de la causación mental.

Todo esto hace dudar seriamente de que el criterio de partir de un espectro de explicaciones que sean dignas de aceptación propuesto por estos autores resulte algo más que una expresión de deseos y no una estrategia programática para enfrentar, con probabilidades ciertas de éxito, el problema de la causación mental.

Por supuesto, Baker y Burge podrían alegar que la opción que favorecen es preferible aun cuando los problemas señalados sean reales, ya que al menos son tratables; mientras que resolver el problema de la causación mental dentro del marco metafísico es imposible. Sin embargo, esto último no ha sido probado; no se ha presentado

${ }^{25} \mathrm{La}$ dificultad en fundamentar el éxito explicativo no implica, sin embargo, la negación del progreso de la disciplina. El descubrimiento de nuevos hechos, el incremento de la capacidad predictiva, el desarrollo tecnológico y praxiológico son dimensiones del progreso de la psicología que necesariamente deben considerarse al momento de valorar si su evolución puede o no calificarse de progresiva.

Crítica, vol. 37, no. 110 (agosto 2005) 
ningún argumento general que demuestre que esto no puede hacerse. Es innegable que el problema es de una complejidad extraordinaria y, quizás, insoluble. No obstante, dado el conjunto de dificultades que hemos señalado sobre el intento de descartar la metafísica, no parece que tengamos a nuestra disposición una estrategia mejor para enfrentarlo. ${ }^{26}$

\section{BIBLIOGRAFÍA}

Baker, Lynn, 1993, "Metaphysics and Mental Causation", en Heil y Mele 1993, pp. 75-95.

Bieri, Peter, 1992, "Trying Out Epiphenomenalism", Erkenntnis, vol. 36, no. 3, pp. 283-309.

Block, Ned, 1990, "Can the Mind Change the World?", en Cynthia Macdonald y Graham Macdonald (comps.), Philosophy of Psychology. Debates on Psychological Explanation, vol. 1, Blackwell, Londres, 1995, pp. 29-59.

Burge, Tyler, 1993, "Mind-Body Causation and Explanatory Practice", en Heil y Mele 1993, pp. 97-120.

Churchland, Paul, 1981, "El materialismo eliminativo y las actitudes proposicionales", en E. Rabossi (comp.), 1995, Filosofía de la mente y ciencia cognitiva, Paidós, Barcelona.

Comesaña, Manuel, 1995, Razón, verdad y experiencia. Un análisis de sus vínculos en la epistemología contemporánea, con especial referencia a Popper, Universidad Nacional de Mar del Plata, Mar del Plata.

Fodor, Jerry, 1987, Psychosemantics, The MIT Press, Cambridge, Mass.

Forster, Malcolm y Eric Saidel, 1994, "Connectionism and the Fate of Folk Psychology: A Reply to Ramsey, Stich and Garon", Philosophical Psychology, vol. 7, no. 4, pp. 437-452.

Glymour, Clark, 1999, "A Mind Is a Terrible Thing to Waste - Critical Notice: Jaegwon Kim, Mind in a Physical World", Philosophy of Science, vol. 66, no. 3, pp. 455-471.

Heil, John y Alfred Mele (comps.), 1993, Mental Causation, Oxford University Press, Oxford.

Horgan, Terence y John Tienson, 1990, "Soft Laws", Midwest Studies in Philosophy, vol. 15, pp. 256-279.

Horgan, Terence y James Woodward, 1985, "Folk Psychology Is Here to Stay", Philosophical Review, vol. 94, pp. 197-226.

Jackson, Frank y Philip Pettit, 1990a, "Program Explanation: A General Perspective", Analysis, vol. 50, pp. 107-117.

${ }^{26}$ Deseo agradecer las valiosas observaciones de los árbitros de Crítica y de Manuel Comesaña, que me permitieron mejorar sustancialmente varios aspectos de este trabajo. 
Jackson, Frank y Philip Pettit, 1990b, "In Defence of Folk Psychology", Philosophical Studies, vol. 59, no. 1, pp. 31-54.

Kim, Jaegwon, 1998, Mind in a Physical World. An Essay on the MindBody Problem and Mental Causation, The MIT Press, Cambridge, Mass.

__ , 1994, "Explanatory Knowledge and Metaphysical Dependence", en E. Villanueva (comp.), Philosophical Issues 5. Truth and Rationality, Ridgeview, Atascadero.

_ 1993, "The Nonreductivist's Troubles with Mental Causation", en Heil y Mele, (comps.), 1993, pp. 00. (Reimpreso en Jaegwon Kim, Supervenience and Mind, Cambridge University Press, Cambridge, 1993.)

— 1990, "Explanatory Exclusion and the Problem of Mental Causation", en E. Villanueva (comp.), Information, Semantics and Epistemology, Blackwell, Cambridge, Mass., 1990. (Reimpreso en Cynthia Macdonald y Graham Macdonald (comps.), Philosophy of Psychology. Debates on Psychological Explanation, vol. 1, Blackwell, Londres, 1995.)

_- 1989a, "Mechanism, Purpose, and Explanatory Exclusion", Philosophical Perspectives 3, Philosophy of Mind and Action Theory, James E. Tomberlin (comp.), Ridgeview, Atascadero. (Reimpreso en Jaegwon Kim, Supervenience and Mind, Cambridge University Press, Cambridge, 1993.)

_ 1989b, "The Myth of Nonreductive Materialism", Proceedings and Addresses of the American Philosophical Association, vol. 63, pp. 3147. (Reimpreso en Jaegwon Kim, Supervenience and Mind, Cambridge University Press, Cambridge, 1993.)

Laudan, Larry, 1986/1977, El progreso y sus problemas. Hacia una teoría del crecimiento científico, trad. Javier López Tapia, Encuentro, Madrid.

Lowe, E. Jonathan, 1999, "Self, Agency and Mental Causation", Journal of Consciousness Studies, vol. 6, nos. 8-9, pp. 225-239.

Marras, Ausonio, 2000, "Critical Notice of Mind in a Physical World" by Jaegwon Kim, Canadian Journal of Philosophy, vol. 30, pp. 137-160.

—_, 1998, "Kim's Principle of Explanatory Exclusion”, Australasian Journal of Philosophy, vol. 76, pp. 439-451.

Newton-Smith, William H., 1987 (1981), La racionalidad de la ciencia, trad. Marco Aurelio Galmarini, Paidós, Barcelona.

O’Brien, Gerard, 1991, "Is Connectionism Common Sense?", Philosophical Psychology, vol. 4, pp. 165-178.

Rabossi, Eduardo, 2004, "La psicología popular y el sentido común. La controversia y los escenarios", en E. Rabossi (comp.), La mente y sus problemas. Temas actuales de filosofía de la psicología, Catálogos, Buenos Aires.

Ramsey, W., S. Stich y J. Garon, 1990, "Connectionism, Eliminativism and the Future of Folk Psychology", Philosophical Perspectives. 4. Philosophy of Mind and Action Theory, James E. Tomberlin (ed.), Ridgeview, Atascadero. (Reimpreso en Cynthia Macdonald y Graham Macdonald (comps.), Connectionism: Debates on Psychological Explanation, Blackwell, Oxford, 1995.) 
Sabatés, Marcelo, 2001, "Varieties of Exclusion", Theoria, vol. 16, no. 40, pp. 13-42.

Stich, Stephen, 1983, From Folk Psychology to Cognitive Science, The MIT Press, Cambridge, Mass.

Thomasson, Amie, 1998, "A Nonreductivist Solution to Mental Causation", Philosophical Studies, vol. 89, pp. 181-195.

Van Fraassen, Bas, 1996/1980, La imagen científica, trad. Sergio Martínez, Paidós/Instituto de Investigaciones Filosóficas-UNAM, México.

Van Gelder, Tim, 1992, "Exposing the Distinction Between Mind and Cognition", en L. Niklasson y M. Boden (comps.), Selected Readings of the Swedish Conference on Connectionism, Ellis Horwood.

Van Gulick, Robert, 1993, "Who's in Charge Here? And Who's Doing All the Work?", en Heil y Mele 1993, pp. 233-256.

Recibido el 9 de agosto de 2004; revisado el 20 de septiembre de 2005; aceptado el 12 de octubre de 2005. 